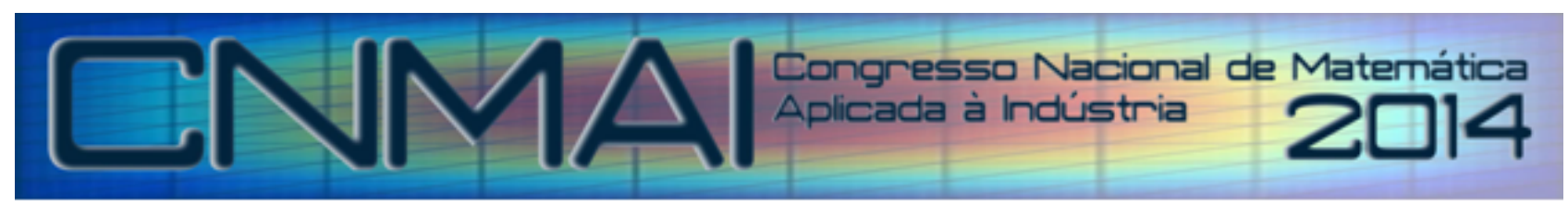

18 a 21 de novembro de 2014, Caldas Novas - Goiás

\title{
RESOLUÇÃO DE PROBLEMAS DE TRANSFERÊNCIA DE CALOR POR CONDUÇÃO USANDO MÉTODOS DE DISCRETIZAÇÃO NO CONTORNO (MESHLESS METHODS)
}

\begin{abstract}
$\mathbf{X X X}, \mathbf{x x x} @ \mathbf{x x} \mathbf{x}^{1}$
${ }^{1} \mathrm{XXXX}$

Resumo. A modelagem de fenômenos físicos e químicos nas diversas áreas da ciência representa uma tarefa desafiadora. Estes modelos são constituídos por equações diferenciais que representam os balanços de massa, energia e quantidade de movimento. Devido a não linearidade que é inerente em tais modelos, a resolução dos mesmo é obtida de forma numérica. Neste cenário, inúmeros métodos têm sido propostos para essa finalidade. Estas abordagens consistem da discretização do contorno bem como do interior da domínio, transformando o problema original em um similar constituído por equações algébricas. Em termos de implementação, pode-se dizer que esta classe de métodos é bem atrativa. Entretanto, como principal desvantagem pode-se citar o alto custo computacional quanto é necessário a sua aplicação várias vezes, como observado em problemas de otimização. Como alternativa para superar esta dificuldade, inúmeros métodos sem malha (Meshless Methods) têm sido propostos. Basicamente, estes consistem da discretização do contorno do domínio, resultando em um sistema algébrico de menor ordem, reduzindo o tempo total de procesamento requerido. Diante do que foi apresentado, a presente contribuição tem por objetivo realizar um estudo comparativo usando o Método de Elementos de Contorno e o Método das Soluções Fundamentais para a resolução de problemas de transferência de calor por condução. Os resultados obtidos são comparados com aqueles obtidos pelo Método de Diferenças Finitas.
\end{abstract}

Palavras-chave: Métodos sem Malha, Simulação, Transferência de Calor por Condução.

\section{INTRODUÇÃO}

A modelagem matemática de processos de transferência de calor por condução é uma linha de pesquisa de grande interesse para a comunidade científica. Isto se deve a quantidade de aplicações que podem ser encontradas em áreas distintas da ciência e engenharia. Dentre estas pode-se citar o seu uso para a determinação de parâmetros termo-físicos ou de condições de contorno. Em muitos problemas de engenharia, parte do contorno do corpo de prova pode ser inacessível às medições diretas ou a presença de sensores pode ser inviável devido às altas temperaturas na região de interesse. Neste caso, a determinação dos parâmetros termo-físicos podem ser obtidos a partir da formulação e resolução de um problema inverso.

Naturalmente, para a aplicação citada, é necessário a resolução de um modelo diferencial parcial inúmeras vezes, o que requer que o mesmo seja resolvido no menor tempo possível. Na literatura, várias abordagens podem ser encontradas para a resolução do modelo que representa o mecanismo de transferência de calor por condução, dentre as quais pode-se citar: $i$ ) o Método das Diferenças Finitas, $i$ ) o Método dos Volumes Finitos e iii) o Método dos Elementos Finitos. Estas técnicas exigem a discretização de pontos internos e externos (contorno) do domínio para a sua aplicação. Dentre os fatores que influenciam o custo de processamento requerido para resolver essa equação diferencial parcial destaca-se o número de pontos de discretizações, que está relacionado com a precisão, e da complexidade da geometria em estudo. Neste caso, quanto maior o refinamento da malha ou quanto maior a complexidade da geometria, maior é o custo de processmento requerido para resolver este modelo, e por consequência, para a resolução de um problema inverso.

Nos últimos anos, o desenvolvimento/aprimoramento das técnicas que não necessitam da discretização de pontos internos do domínio (métodos meshless ou meshfree) para a resolução da equação diferencial parcial tem atraíndo a atenção da comunidade científica. Basicamente, estas estratégias fundamentam-se, a partir da definição de uma equação integral e/ou de uma solução fundamental, em reescrever o problema original definido em todo o domínio, em um equivalente que 
considera a discretrização em pontos definidos nos contornos. Dentre estas técnicas, o Método dos Elementos de Contorno (MEC) e Método das Soluções Fundamentais (MSF) configuram-se como interessantes alternativas para a resolução de equações diferenciais parciais.

Diante do que foi apresentado, este trabalho tem por objetivo a aplicação do MEC e do MSF para a resolução da equação de transferência de calor por condução. Este trabalho é estruturado conforme segue. As Seções 2 e 3 trazem a caracterização do problema de interesse e uma breve descrição dos métodos numéricos para resolução de EDPs, respectivamente. Na Seção 4 são apresentados os resultados obtidos com a aplicação das técnicas propostas. Finalmente, as conclusões e perspectivas para trabalhos futuros são descritas na última seção.

\section{O PROBLEMA DE INTERESSE: TRANSFERÊNCIA DE CALOR POR CONDUÇÃO}

Considere uma placa plana de dimensões $L(0,40 \mathrm{~m}) \times H(0,80 \mathrm{~m})$ com as suas superfícies mantidas a diferentes temperaturas conforme apresentado na Fig. 1 (Braga Filho, 2004).

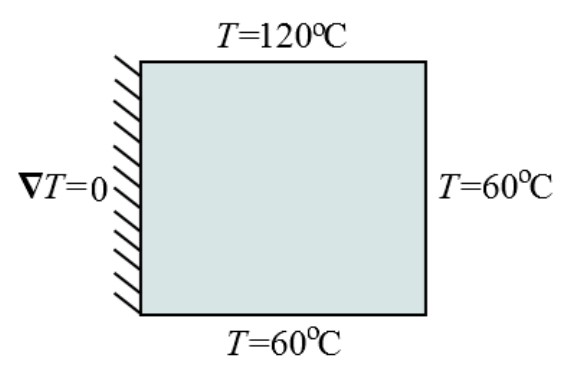

Figura 1. Definição do problema de condução.

Considerando regime permanente, que não existe termo fonte e aproximação bidimensional, o problema matemático é formulado como:

$$
\begin{gathered}
\frac{\partial^{2} T}{\partial x^{2}}+\frac{\partial^{2} T}{\partial y^{2}}=0 \\
\left.\frac{\partial T}{\partial x}\right|_{(x=0, y)}=0 \\
\left.T\right|_{(x=L, y)}=60^{\circ} \mathrm{C} \\
\left.T\right|_{(x, y=0)}=60^{\circ} \mathrm{C} \\
\left.T\right|_{(x, y=H)}=120^{\circ} \mathrm{C}
\end{gathered}
$$

Braga Filho (2004) demonstrou que a solução analítica para o problema formulado é dada por:

$$
T(x, y)=60+120 \sum_{n=0}^{\infty} \frac{(-1)^{n}}{\lambda_{n} L} \frac{\sinh \left(\lambda_{n} y\right)}{\sinh \left(\lambda_{n} H\right)} \cos \left(\lambda_{n} x\right)
$$

onde $n$ é o número de pontos considerados no somatório e o parâmetro $\lambda_{n}$ é definido como:

$$
\lambda_{n}=\frac{(2 n+1) \pi}{2 L}, n=0,1, \ldots, \infty
$$

\section{MÉTODOS NUMÉRICOS PARA A RESOLUÇÃO DE EDP'S}

O desenvolvimento de métodos numéricos para a resolução de problemas modelados por Equações Diferenciais Parciais (EDP's) é uma linha de pesquisa de grande interesse devido às inúmeras aplicações que podem ser realizadas. Tradicionalmente, estas EDP's são resolvidas utilizando-se métodos de discretização de pontos internos e externos do domínio de interesse. Dentre estes, destacam-se o Método das Diferenças Finitas (MDF) e o Método dos Elementos Finitos, que diferenciam-se quanto ao processo de discretização da geometria do problema, conforme apresentado na Fig. 2.

No Método das Diferenças Finitas o domínio é dividido em um número finito de pontos nos quais serão determinados os valores da variável dependente. A qualidade de solução, isto é, a sua precisão esta diretamente relacionada com o número de pontos considerados na malha. Todavia, quanto maior o grau de refinamento maior será o sistema de equações a ser resolvido, e por consequência, maior é o esforço computacional requerido. Como principal vantagem pode-se citar a sua concepção conceitual simples e a sua facilidade de implementação. Entretanto, a sua aplicabilidade está diretamente relacionada com a geometria do problema, isto é, não sendo facilmente adaptado para geometrias mais complexas. 


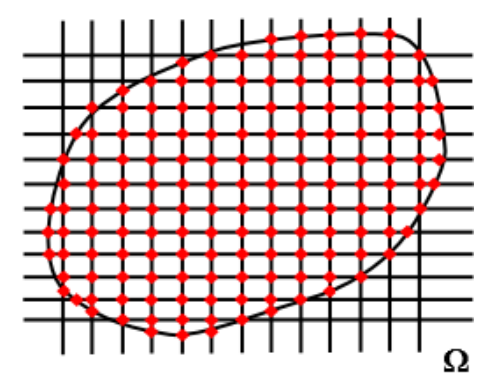

(a) Método das Diferenças Finitas

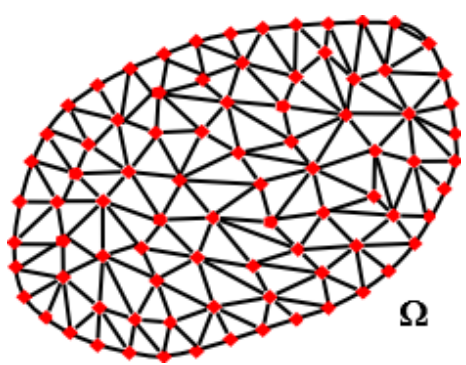

(b) Método dos Elementos Finitos.

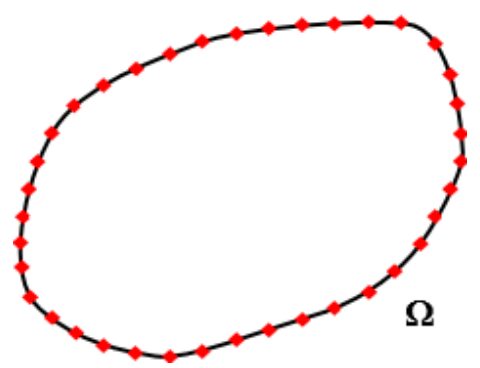

(c) Meshless Methods.

Figura 2. Interpretação Geométrica dos Métodos Com e Sem Malha.

Já no Método dos Elementos Finitos o domínio é dividido em elementos geométricos sendo que a variável dependente é obtida nos vértices de cada um destes elementos. Assim como no Método das Diferenças Finitas, a sua precisão está diretamente relacionada com o grau de refinamento da malha, o que implica na dimensão do sistema algébrico a ser resolvido. Como principal vantagem do Método dos Elementos Finitos pode-se citar o tratamento de geometrias complexas, além de permitir um maior refinamento da malha em regiões particulares do domínio de interesse.

Apesar da grande aplicabilidade dos métodos citados, a sua utilização em problemas com geometrias complexas pode não ser computacionalmente viável, já que estes exigem o refinamento da malha, e por consequência, um elevado esforço computacional. Em se tratando da resolução de problemas de otimização sujeitos a restrições diferenciais, exige-se a avaliação, a cada iteração, destas equações. Neste caso, o custo computacional é demasiado elevado. Para superar tal dificuldade, nas últimas décadas, inúmeros esforços têm sido dedicados ao desenvolvimento/aprimoramento de Métodos sem Malha (Meshless/Meshfree Methods), isto é, métodos que exigem a discretização apenas de pontos localizados no contorno e não de pontos internos no domínio. Neste caso, o contorno é particionado em segmentos ao longo dos quais deve-se determinar o valor da variável dependente ou de sua derivada na direção normal (dependendo de qual condição de contorno é aplicada em cada elemento). A partir dos valores obtidos sobre o contorno, pode-se determinar o valor da variável dependente em qualquer ponto no interior do domínio. A grande vantagem destes métodos é que eles necessitam somente da discretização dos pontos localizados no contorno do domínio de interesse, e por consequência, a obtenção de um sistema algébrico de menor dimensão. Todavia, ressalta-se que o desenvolvimento matemático de tais métodos é muito mais sofisticado que as abordagens que fazem uso da discretização de pontos internos no domínio, podendo gerar sistemas mal condicionados. Além disso, a sua aplicação está diretamente relacionada ao conhecimento de uma solução característica fundamental, e que é diferente para cada tipo de problema (Ang, 2007; Alves e Chen, 2005; Alves e Valtchev, 2005).

Diante do que foi apresentado, esta seção tem por objetivo apresentar, de forma simplificada, o desenvolvimento matemático dos Método dos Elementos de Contorno (MEC) e do Método das Soluções Fundamentais (MSF).

\subsection{Método dos Elementos de Contorno}

O termo MEC (Boundary Element Method) foi empregado pela primeira vez por Brebbia e Dominguez (1977). Desde as suas primeiras aplicações, este tem-se configurado como uma interessante estratégia para o tratamento de problemas com geometrias e condições de contorno complexas. Mesmo sendo considerada uma abordagem relativamente nova (em termos de aplicações), o MEC tem-se desenvolvido/aprimorado nas últimas décadas. A principal vantagem do MEC é que ele necessita que somente o contorno do domínio seja discretizado, o que reduz significativamente o esforço computacional em relação as técnicas clássicas para a resolução de equações diferenciais parciais (Ang, 2007). Basiciamente, no MEC é realizado a divisão do contorno em segmentos ao longo dos quais deve-se determinar o valor da variável dependente ou de sua derivada na direção normal, dependendo de qual a condição de contorno é aplicada em cada elemento, resultando desta forma, a redução da dimensão do problema (Menin, 2009).

O MEC objetiva transformar uma equação diferencial que atua sobre todo o domínio $\Omega$ em uma equação integral que atua somente sobre o contorno $\partial \Omega$ do domínio, conforme apresentado na Fig. 3.

Esta transformação é feita através da chamada solução recíproca, que é uma relação entre duas soluções particulares quaisquer, $\Phi$ e $\phi$, da equação diferencial parcial e de suas derivadas na direção normal, $\partial \Phi / \partial n$ e $\partial \phi / \partial n$, sobre o contorno do domínio $\partial \Omega$, e que pode ser calculada, por exemplo, para $\phi$ como

$$
\frac{\partial \phi}{\partial n}=n_{x} \frac{\partial \phi}{\partial x}+n_{y} \frac{\partial \phi}{\partial y}
$$

onde $n_{x}$ e $n_{y}$ são as componentes $x$ e $y$ do vetor normal unitário. 


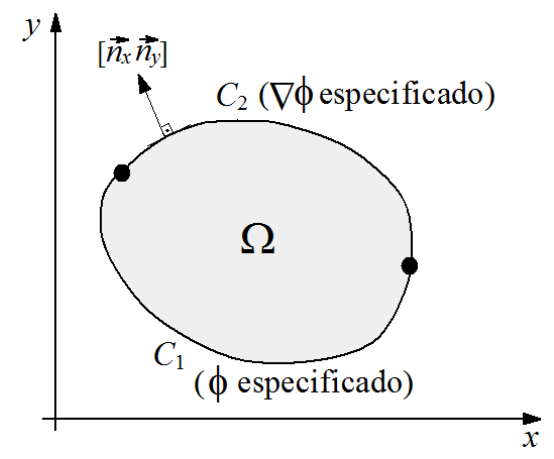

Figura 3. Definição das condições de contorno para a aplicação do MEC.

A obtenção da solução recíproca pode ser feita através do teorema da divergência do cálculo diferencial e integral. Para essa finalidade, seja $\Phi$ e $\phi$ duas soluções da equação de Laplace

$$
\begin{aligned}
& \frac{\partial^{2} \Phi}{\partial x^{2}}+\frac{\partial^{2} \Phi}{\partial y^{2}}=0 \\
& \frac{\partial^{2} \phi}{\partial x^{2}}+\frac{\partial^{2} \phi}{\partial y^{2}}=0
\end{aligned}
$$

Se multiplicarmos a primeira equação por $\phi$ e a segunda equação por $\Phi$ e tomarmos a diferença deste resultado, obtêm-se

$$
\frac{\partial}{\partial x}\left(\phi \frac{\partial \Phi}{\partial x}-\Phi \frac{\partial \phi}{\partial x}\right)+\frac{\partial}{\partial y}\left(\phi \frac{\partial \Phi}{\partial y}-\Phi \frac{\partial \phi}{\partial y}\right)=0
$$

e que pode ser integrada no domínio $\Omega$

$$
\iint_{\Omega}\left(\frac{\partial}{\partial x}\left(\phi \frac{\partial \Phi}{\partial x}-\Phi \frac{\partial \phi}{\partial x}\right)+\frac{\partial}{\partial y}\left(\phi \frac{\partial \Phi}{\partial y}-\Phi \frac{\partial \phi}{\partial y}\right)\right) d x d y=0
$$

A aplicação do teorema da divergência converte a integral de área em uma integral de contorno $(\partial \Omega)$

$$
\int_{\partial \Omega}\left(\left(\phi \frac{\partial \Phi}{\partial x}-\Phi \frac{\partial \phi}{\partial x}\right) n_{x}+\left(\phi \frac{\partial \Phi}{\partial y}-\Phi \frac{\partial \phi}{\partial y}\right) n_{y}\right) d s(x, y)=0
$$

Sabendo que para a equação de Laplace a solução fundamental é:

$$
\Phi(x, y, \xi, \eta)=\frac{1}{2 \pi} \ln \left((x-\xi)^{2}+(y-\eta)^{2}\right)
$$

onde $\xi$ e $\eta$ é um ponto coordenado genérico.

Assim, pode-se mostrar que o valor de $\phi(x, y, \xi, \eta)$ no contorno $\partial \Omega$ é dado por:

$$
\lambda(\xi, \eta) \phi(\xi, \eta)=\int_{\partial \Omega} \phi(x, y) \frac{\partial \Phi(x, y, \xi, \eta)}{\partial n}-\Phi(x, y, \xi, \eta) \frac{\partial \phi(x, y)}{\partial n} d s(x, y)
$$

onde $\lambda(\xi, \eta)$ é uma constante definida a partir do domínio. Se o ponto $(\xi, \eta)$ não pertence a $\Omega, \lambda(\xi, \eta)$ é igual a 0 ; se o ponto $(\xi, \eta)$ pertence a $\Omega, \lambda(\xi, \eta)$ é igual a $1 / 2$, caso contrário $\lambda(\xi, \eta)$ é igual a 1 .

A descrição matemática detalhada sobre o desenvolvimento apresentado pode ser encontrado em Ang (2007).

\subsection{Método das Soluções Fundamentais}

O Método das Soluções Fundamentais (MSF), também conhecido como Método Desingularizado (Desingularized Method) ou Método da Simulação de Carga (Charge Simulation Method) ou Método da Superposição (Superposition Method), foi publicado originalmente por Kupradze e Aleksidze em 1964 (Fairweather et al., 2003; Alves e Chen, 2005; Alves e Valtchev, 2005; Dong et al., 2007).

A ideia principal do MSF consiste em aproximar a solução do problema através de uma combinação linear de soluções fundamentais com respeito a certas singularidades (também chamadas de pontos-fonte) que estão localizadas em uma fronteira fictícia (geralmente um círculo) fora do domínio, conforme apresntado na Fig. 4. 


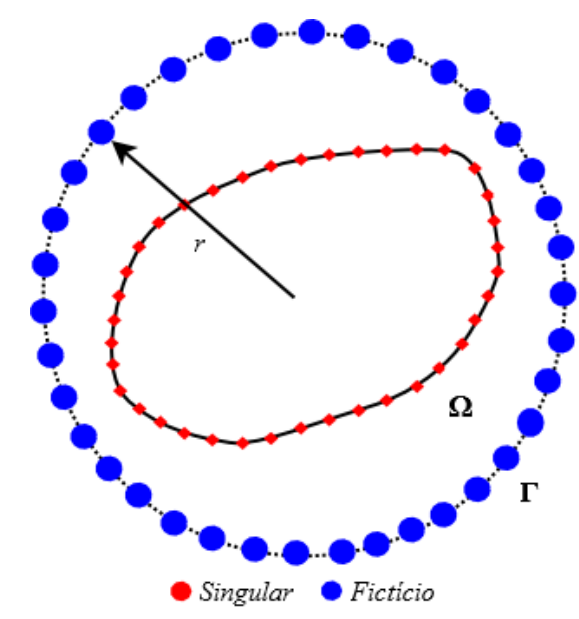

Figura 4. Localização das singularidades em uma fronteira fictícia no MSF.

Como principais vantagens do MSF pode-se citar (Fairweather et al., 2003; Alves e Chen, 2005; Alves e Valtchev, 2005; Dong et al., 2007): $i$ ) não requer uma discretização elaborada do contorno, $i i$ ) não há necessidade de integrações sobre o contorno, $i i i$ ) a solução no interior do domínio é obtida sem a necessidade de quadraturas extras, $i v$ ) é de fácil implementação. Por outro lado, como principais desvantagens pode-se citar: $i$ ) a necessidade do conhecimento de um solução fundamental referente a equação diferencial em questão e $i i$ ) os sistemas lineares provenientes da discretização via MSF tendem a ser mal condicionados.

Uma solução fundamental $g(x, y)$ é a solução da equação diferencial parcial homogênea

$$
£(u)=0 \text { em } \Omega
$$

em todo o domínio $\Omega$ que não contenha o ponto singular $y$ de $g(x, y)$. Se $\Omega \subset \mathbb{R}^{d}$, com $d=2,3$, é um domínio conexo aberto não-vazio limitado, com um contorno suficientemente regular $\delta \Omega=\Gamma$ e $f^{\Gamma}$ é uma função conhecida, então a condição de contorno

$$
u=f^{\Omega} \text { em } \Omega
$$

define, juntamente com a Eq. 17, o problema homogêneo de valor de contorno de Dirichlet. No método clássico, utiliza-se uma integral de contorno para se representar $u$ em termos do potencial de duas camadas

$$
u(x)=\int_{\Gamma} \frac{\partial g(x, y)}{\partial n} \sigma(y) d \gamma(y), x \in \Gamma
$$

onde $n$ é o vetor normal para em $y \in \Gamma, \sigma$ é a densidade desconhecida e $g(x, y)$ é a solução fundamental com a singularidade $x$ localizada no contorno. Assim, o problema de valor de contorno se reduz a uma equação integral de contorno singular a ser resolvida para $\sigma$ no contorno

$$
f^{\Gamma}(x)=\int_{\Gamma} \frac{\partial g(x, y)}{\partial n} \sigma(y) d \gamma(y), x \in \Gamma
$$

Para representar $u$ por um potencial de uma camada para a obtenção da solução das Eqs. 16 e 17 através da seguinte relação:

$$
u(x)=\int_{\Gamma} g(x, y) \sigma(y) d \gamma(y), x \in \Gamma
$$

Para determinar a distribuição de densidades $\sigma$ no contorno deve ser considerado:

$$
\int_{\Gamma} g(x, y) \sigma(y) d \gamma(y)=f^{\Gamma}(x)
$$

De modo a diminuir as dificuldades de integrais singulares, é possível mover o domínio de integração para fora de $\Omega$ de forma a obter uma solução homogênea. Desse modo, surgem funções de interpolação do tipo

$$
u(x)=\int_{\hat{\Gamma}} g(x, y) \sigma(y) d \gamma(y), x \in \Gamma
$$

onde o contorno fictício $\hat{\Gamma}$ é o contorno do domínio $\hat{\Omega}$ e que contém $\Omega$. Desde que a Eq. 22 automaticamente satisfaça a Eq. 16, é necessário apenas satisfazer a condição de contorno da Eq. 17 por meio das funções de interpolação da Eq. 22. Essas condições nos levam a

$$
\int_{\hat{\Gamma}} g(x, y) \sigma(y) d \gamma(y)=f^{\Gamma}, x \in \Gamma
$$


onde a distribuição de densidades $\sigma$ é determinada no contorno $\hat{\Gamma}$ ao invés de $\Gamma$. Uma vez determinado $\sigma$, a solução $u$ pode ser obtida.

Neste caso, observa-se que nenhuma integração foi necessária, com acontece no MEC, bastando apenas se superpor um número de soluções fundamentais para se obter uma boa aproximação dos valores de contorno.

\section{APLICAÇÕES}

Para fins de aplicação da metodologia proposta neste trabalho, a seguir são apresentados dois estudos de caso fundamentados no problema definido na seção 2, a saber, o primeiro sem cavidade e o segundo com cavidade. Neste cenário, foram consideradas algumas configurações para cada um dos métodos, conforme descritos a seguir:

- Solução Analítica: para a avaliação da Eq. 6, foram considerados 20 termos no somatório, o que garante uma boa precisão (Braga Filho, 2004).

- MDF: para essa análise foram considerados uma aproximação de segunda ordem para as direções $x$ e $y$, além de diferentes níveis de discretização para fins de comparação, conforme apresentado a seguir.

- MEC: foram considerados diferentes valores para o número de pontos de discretização do contorno.

- MSF: foram considerados diferentes valores para o tamanho do raio do círculo fictício, bem como do número de pontos de discretização do contorno e 25 pontos de discretização no círculo fictício.

Em todos os estudos de casos foram considerados 100 pontos internos onde avaliou-se a solução numérica, bem como a solução analítica para fins de comparação.

\subsection{Domínio Sem Cavidade}

A Tab. 1 apresenta a comparação entre os resultados obtidos pelos métodos MDF, MEC e MSF para o estudo de caso sem cavidade.

Tabela 1. Comparação entre os resultados obtidos pelos MDF, MEC e MSF para o estudo de caso sem cavidade.

\begin{tabular}{c|c|c|c|c}
\hline & Raio & Número de Elementos & Dimensão & Erro (\%) \\
\hline MDF & - & $10 / 25$ & $121 / 676$ & $4,08 / 1,89$ \\
MEC & - & $121 / 676$ & $121 / 676$ & $2,02 / 1,75$ \\
& 1,2 & $121 / 676$ & $121 / 676$ & $2,12 / 1,72$ \\
MSF & 2,0 & $121 / 676$ & $121 / 676$ & $2,11 / 1,71$ \\
& 5,0 & $121 / 676$ & $121 / 676$ & $2,10 / 1,70$ \\
\hline
\end{tabular}

Nesta tabela observa-se a mesma qualidade, em termos do erro comentido, de ambas as estratégias que não fazem uso da discretização de pontos internos do domínio. Além disso, as mesmas tem menor erro em comparação com o MDF para diferentes dimensões do sistema linear resultante da discretização. Em relação ao raio utilizado no MSF, para o estudo de caso considerado, o aumento deste parâmetro não implicou em reduções significativas do erro cometido.

A Fig. 5 apresenta o perfil de temperatura correspondente ao problema sem cavidade obtido via aplicação do MEC (para os parâmetros apresentados na Tab. 1).

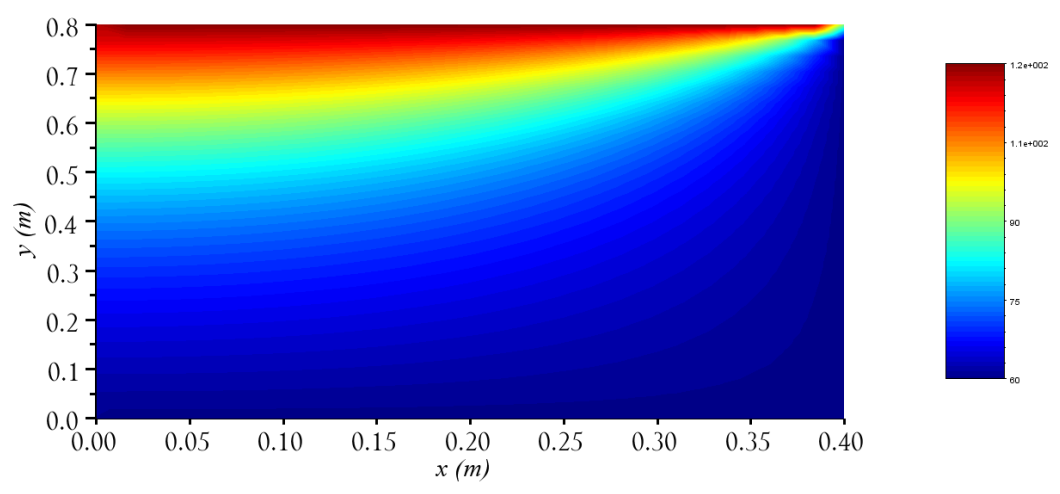

Figura 5. Perfil de temperatura para o problema de condução de calor sem cavidade.

A Fig. 6 apresenta o erro comentido usando cada metodologia em função da dimensão do sistema resultante do processo de discretização para o problema sem cavidade. Nesta, observa-se, como esperado, que o aumento da dimensão do sistema linear resulta na redução do erro cometido. Todavia, o perfil do erro cometido é similiar para o MEC e para o MSF e bem inferior, pelo menos para sistemas com menores dimensões, em relação o MDF. 


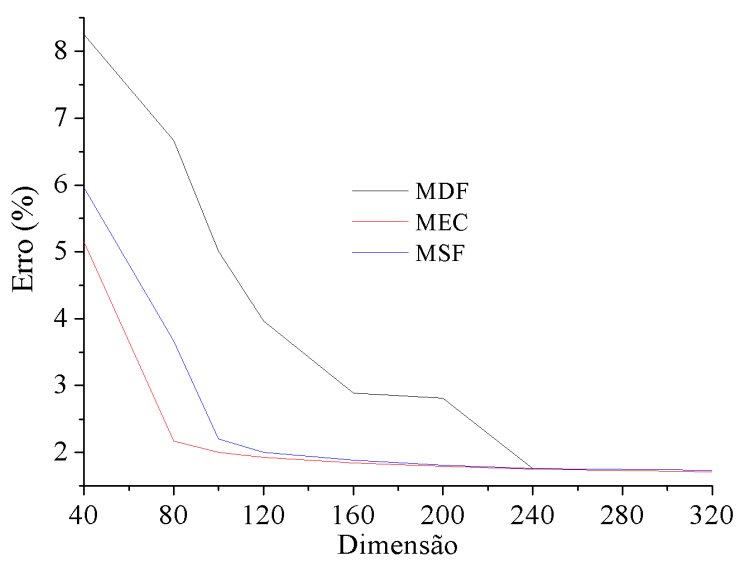

Figura 6. Erro versus dimensão do sistema para o problema sem cavidade.

\subsection{Domínio Com Cavidade}

O problema com cavidade é o mesmo definido pela Fig. 1, entrento com a presença de uma cavidade, conforme apresentado na Fig. 7.

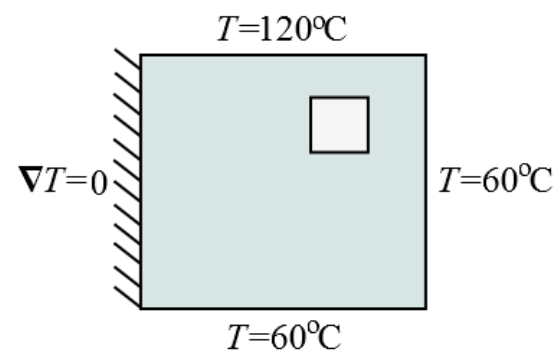

Figura 7. Definição do problema de condução com cavidade.

Neste caso, as dimensões da cavidade são: $0,35 \leq x \leq 0,35$ e $0,5 \leq y \leq 0,55$. As condições de contorno consideradas na cavidade foram definidas a partir da avaliação da temperatura dada pela solução analítica (Eq. 6).

Na Tab. 2 são apresentados a comparação entre os resultados obtidos pelos métodos MDF, MEC e MSF para o estudo de caso com cavidade.

Tabela 2. Comparação entre os resultados obtidos pelos MDF, MEC e MSF para o estudo de caso com cavidade.

\begin{tabular}{c|c|c|c|c}
\hline & Raio & Número de Elementos & Dimensão & Erro (\%) \\
\hline MDF & - & $10 / 25$ & $121 / 676$ & $4,08 / 1,73$ \\
MEC & - & $121 / 676$ & $121 / 676$ & $2,27 / 1,72$ \\
& 1,2 & $121 / 676$ & $121 / 676$ & $2,16 / 1,70$ \\
MSF & 2,0 & $121 / 676$ & $121 / 676$ & $2,14 / 1,70$ \\
& 5,0 & $121 / 676$ & $121 / 676$ & $2,15 / 1,68$ \\
\hline
\end{tabular}

Assim como verificado para o estudo de caso anterior, observa-se a mesma qualidade, em termos do erro comentido, de ambas as estratégias que não fazem uso da discretização de pontos internos do domínio. Em comparação com o MDF, o MEC e o MSF apresentam nemor erro.

A Fig. 8 apresenta o perfil de temperatura correspondente ao problema com cavidade obtido via aplicação do MEC (para os parâmetros apresentados na Tab. 2). 


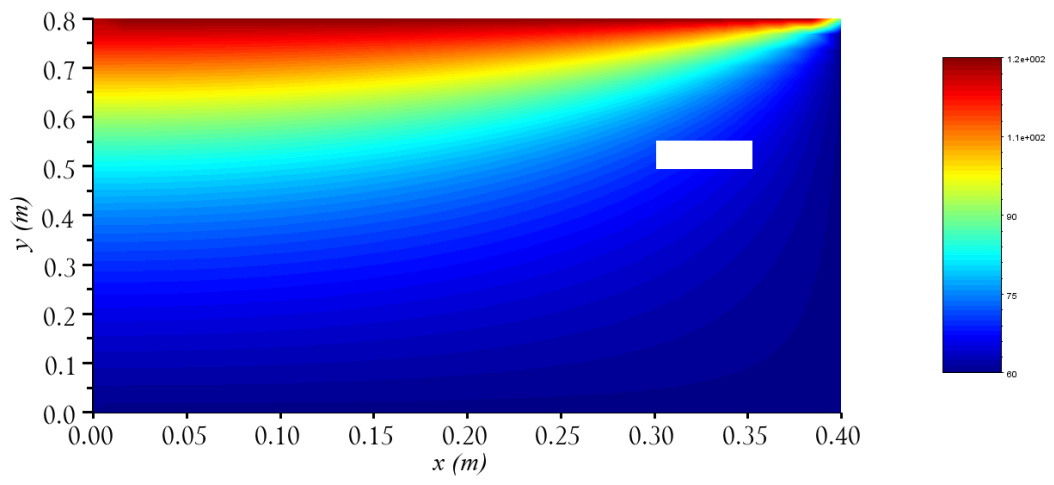

Figura 8. Perfil de temperatura para o problema de condução de calor com cavidade.

A Fig. 9 apresenta o erro comentido usando cada metodologia em função da dimensão do sistema resultante do processo de discretização para o problema com cavidade. Como esperado, constata-se que o aumento da dimensão do sistema linear resulta na redução do erro cometido, já que isto resulta em refinamento da malha. Todavia, assim como observado para o problema sem cavidade, o perfil do erro cometido é similiar para ambas as estratégias sem o uso de malha (MEC e MSF) e bem inferior, pelo menos para sistemas com menores dimensões, em relação o MDF.

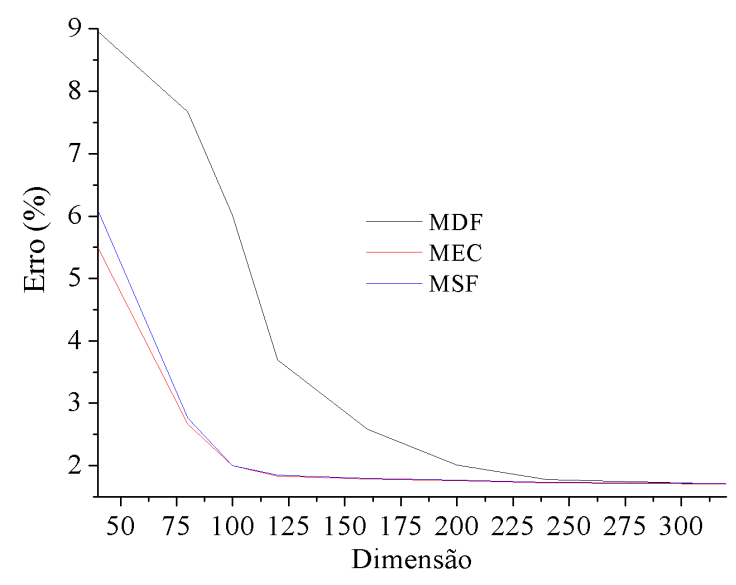

Figura 9. Erro versus dimensão do sistema para o problema com cavidade.

\section{CONCLUSÕES}

O presente trabalho teve por objetivo avaliar o desempenho do MEC e do MSF para a simulação do problema de transferência de calor por condução. Para essa finalidade, foi considerado um problema com geometria simples e que possui solução analítica para fins de comparação. Os resultados encontrados pelo MEC e pelo MSF foram comparados com aqueles obtidos pelo MDF para diferentes níveis de discretização e para problemas com e sem a presença de uma cavidade. A aplicação em dois estudos de caso demonstraram que ambos os métodos sem malha foram capazes de estimar satisfatoriamente o perfil de temperatura na placa em realção ao MDF. Neste contexto, ambos os métodos sem malha configuraram-se como uma interessante estratégia a ser empregada para a resolução do problema direto quando da formulação de um problema inverso. Em termos do custo benefício, verifica-se que estes métodos tem melhor precisão as custas de um menor esforço computacional (dimensão do sistema linear) quando comparado com o tradicional MDF.

Como continuação deste trabalho pretende-se formular o problema inverso para fins de estudo da determinação de tumores através da técnica de termografia.

\section{AGRADECIMENTOS}

O autor agradece a FAPEMIG e ao CNPq pelo apoio financeiro para a participação no congresso. 


\title{
REFERÊNCIAS
}

Ang, W. T., 2007. A Beginner's Course in Boundary Element Methods. Universal Publishers Boca Raton, USA, 253 pages.

Alves, C. J. S., Chen, C. S., 2005. A New Method of Fundamental Solutions Applied to Nonhomogeneous Elliptic Problems. Advances in Computational Mathematics, vol. 23, pp. 125-142.

Alves, C. J. S., Valtchev, C. S., 2005. Numerical Comparison of Two Meshfree Methods for Acoustic Wave Scattering. Engineering Analysis with Boundary Elements, vol. 29, pp. 371-382.

Braga Filho, W., 2004. Transmissão de Calor. Editora Thonson, Primeira Edição. 428p.

Brebbia, C. A., Dominguez, J., 1977. Boundary Element Methods for Potential Problems. Applied Mathematical Modelling, vol. 1, pp. 372-378.

Chan, C. L., 1991. Boundary Element Method Analysis for the Bioheat Transfer Equation. Journal of Biomechanical Engineering, vol. 1, pp. 358-365.

Dong, C. F., Sun, F. Y., Meng, B. Q., 2007. A Method of Fundamental Solutions for Inverse Heat Conduction Problems in an Anisotropic Medium. Engineering Analysis with Boundary Elements, vol. 31, pp. 75-82.

Fairweather, G., Karageorghis, A., Martin, P. A., 2003. The Method of Fundamental Solutions for Scattering and Radiation Problems. Engineering Analysis with Boundary Elements, vol. 27, pp. 759-769.

Menin, O. H., 2009. Método dos Elementos de Contorno para Tomografia em Impedância Elétrica. Dissertação de Mestrado, Faculdade de Filosofia, Ciências e Letras de Ribeirão Preto, Programa de Física Aplicada à Medicina e Biologia.

\section{RESPONSABILIDADE AUTORAL}

O autor é o único responsável pelo conteúdo deste trabalho.

\section{SOLUTION OF CONDUCTION HEAT TRANSFER PROBLEMS USING BOUNDARY DISCRETIZATION METHODS}

\author{
$\mathbf{X X X}, \mathbf{x x x} \times \mathbf{x x} \mathbf{x}^{1}$
}

${ }^{1} \mathrm{XXXX}$

\begin{abstract}
The modeling of physical and chemical phenomena is a challenging task in various fields of science. These models consist of differential equations representing the mass, energy and momentum balances. Due to nonlinearity that is inherent in such models, its resolution is obtained numerically. In this context, various methods have been proposed for this purpose. These approaches consist of discretization of boundary and interior points of domain, transforming the original problem in a similar consisting of algebraic equations. In terms of implementation, this class of methods is very attractive. However, the main disadvantage is the computational cost associated to solve problems with complex geometries, as observed in optimization problems. To overcome this difficulty, meshless methods have been proposed. Basically, these consists of discretization of boundary, resulting in an algebraic system of lower order, reducing the total time required. In this contribution, a comparative study using the Boundary Element Method and the Method of Fundamental Solutions for solve conduction heat transfer problems is realized. The results are compared with those obtained by the Finite Differences Method.
\end{abstract}

Keywords: Meshless Methods, Simulation, Conduction Heat Transfer Problems. 